\title{
Bifosfonatos en oncología
}

\author{
P. KHOSRAVI SHAHI, V. DÍAZ MUÑOZ DE LA ESPADA \\ Servicio de Oncología Médica. Hospital General Universitario Gregorio Marañón. \\ Madrid
}

BIPHOSPHONATES ON ONCOLOGY

\begin{abstract}
RESUMEN
Los bifosfonatos son compuestos sintéticos análogos de la molécula de pirofosfato endógeno. El mecanismo de acción de los bifosfonatos consiste en la inhibición de la activación o reclutamiento de los osteoclastos (efecto antirresortivo). La biodisponibilidad por vía oral es de 1$2 \%$, lo que unido a sus efectos adversos sobre el tracto digestivo (sobre todo irritación esofágica), lleva a que su utilidad por esa vía sea escasa en el campo de la oncología. Los bifosfonatos se han usado en la enfermedad de Paget óseo, en la osteoporosis, y en otras situaciones como en la enfermedad ósea asociado al trasplante de órganos fuera del campo de la oncología. Mientras que en el campo de la oncología los bifosfonatos se emplean en el tratamiento de la hipercalcemia tumoral, en la prevención y tratamiento de los eventos óseos asociados a la metástasis óseas y en la prevención de la osteoporosis asociada al cáncer de mama. Según las recomendaciones de la American Society of Clinical Oncology (ASCO), la indicaciones aceptadas para el empleo bifosfonatos incluyen la hipercalcemia tumoral y los eventos óseos asociados al cáncer de mama y mieloma múltiple.
\end{abstract}

PALABRAS CLAVE: Bifosfonatos. Oncología. Hipercalcemia tumoral. Cáncer mama. Osteoporosis.

\begin{abstract}
Biophosphonates are analogues of pyrophosphate. The mecanism of action of bisphosphates is the inhibition of osteoclast activation (antiresorptive mechanism). The oral bioavailability of biophosphonates is only about 1-2\%, and because of gastrointestinal side effects (mainly esophageal irritation), oral agents are less useful in oncology. Biophosphonates are used for the treatment of Paget's disease of bone, the prevention of osteoporosis, and in another clinical scenarios as the prevention of bone disease after organ transplantation. In clinical oncology biophosphonates are used for the treatment of hypercalcemia of malignancy, prevention and treatment of bone events related to bone metastases, and in the prevention of osteoporosis related to breast cancer. Acording to American Society of Clinical Oncology (ACO) guidlines, biophosphonates should be used in hypercalcemia of malignancy and bone events related to metastases of breast cancer and multiple myeloma.
\end{abstract}

KEY WORDS: Bisphophonates. Oncology. Hypercalcemia of malignancy. Breast cancer. Osteoporosis.

Khosravi Shahi P, Díaz Muñoz de la Espada V. Bifosfonatos en oncología. An Med Interna (Madrid) 2005; 22: 544-547.

\section{INTRODUCCIÓN}

Los bifosfonatos son compuestos sintéticos análogos de la molécula de pirofosfato endógeno, en la que la estructura $\mathrm{P}$ O-P ha sido sustituida por la P-C-P; y la presencia de este doble grupo fosfónico le confiere particular resistencia a la hidrólisis.

El mecanismo de acción de los bifosfonatos consiste en la inhibición de la activación o reclutamiento de los osteoclastos mediado por PTH, PTHrp, calcitriol, prostaglandinas y citoci- nas (IL-1, TNF) (1,2). La biodisponibilidad por vía oral es de $1-2 \%$, lo que unido a sus efectos adversos sobre el tracto digestivo (sobre todo irritación esofágica), lleva a que su utilidad por esa vía sea escasa en el campo de la oncología $(3,4)$.

Los bifosfonatos fuera del mundo de la oncología se emplean en el tratamiento de la enfermedad de Paget óseo (5), en el tratamiento y prevención de la osteoporosis (6), y en otras situaciones como en la prevención de la enfermedad ósea tras el trasplante de órganos (7), osteogénesis imperfecta y enfermedad de McCune-Albright (8). 
En el campo de la oncología los bifosfonatos se emplean en el tratamiento de la hipercalcemia tumoral (9), en la prevención y tratamiento de los eventos óseos asociados a la metástasis óseas (10) y en la prevención de la osteoporosis asociada al cáncer de mama.

PAPEL DE LOS BIFOSFONATOS EN LA HIPERCALCEMIA TUMORAL

La presencia de hipercalcemia tumoral supone un pronostico infausto, ya que aproximadamente un 50\% de los pacientes fallecen en los 30 días siguientes (11). La hipercalcemia tumoral puede tener lugar por varios mecanismos de producción diferentes (12):

1. Asociado a lesiones osteolíticas: supone un $20 \%$ de los casos, y se debe a un incremento marcado de la resorción ósea por los osteoclastos mediado por citocinas y PTHrp (péptido relacionado con la $\mathrm{PTH}$ ) en las áreas vecinas a las células tumorales. En estos casos las metástasis óseas suelen ser múltiples y extensas. Es la hipercalcemia tumoral que se produce en el seno del mieloma múltiple, cáncer de mama y algunos linfomas (13)

2. Humoral: es el mecanismo de producción más frecuente, constituyendo el $80 \%$ de los casos. En estos casos las metástasis óseas suelen estar ausentes o ser mínimas. Este tipo de hipercalcemia tumoral está producido por la secreción neoplásica de PTHrp (12). El PTHrp incrementa la resorción ósea, y potencia la retención de calcio a nivel renal. La hipercalmia humoral es propia de los carcinomas escamosos (esófago, pulmón, cérvix, cabeza y cuello), y de otras muchas neoplasias como cáncer renal, ovárico, endometrial y cáncer de mama (14).

3. Los otros mecanismos de producción son muy infrecuentes $(<1 \%)$ e incluyen la secreción de 1,25(OH)2 vitamina $\mathrm{D}$ que suele darse en algunos linfomas; y la secreción ectópica de PTH.

Los bifosfonatos son considerados por la Food and Drug Administration (FDA) como los fármacos de elección en el tratamiento de la hipercalcemia tumoral, siendo aceptados para dicha indicación pamidronato y zolendronato por vía intravenosa $(1,12)$. La indicación del tratamiento con bifosfonatos intravenosos suele marcarla unas cifras de calcemia superior a $12 \mathrm{mgdl}$ yo la presencia de síntomas (15). El zolendronato proporciona una disminución más rápida y efectiva del calcio sérico que el pamidronato y una respuesta de mayor duración, por lo que se puede considerar el tratamiento de elección de la hipercalcemia tumoral (16).

El ibandronato podría ser tan efectiva como el pamidronato en el tratamiento de la hipercalcemia tumoral.

\section{PAPEL DE LOS BIFOSFONATOS EN EL TRATAMIENTO Y LA} PREVENCIÓN DE LOS EVENTOS ÓSEOS ASOCIADOS AL CÁNCER

Los eventos óseos asociados al cáncer incluyen el dolor óseo, la hipercalcemia tumoral, las fracturas patológicas y la compresión radiculo-medular.

Los bifosfonatos tienen un beneficio modesto en el control del dolor óseo causado por metástasis óseas (17), lo que añadido a su efecto analgésico tardío (inicio de efecto en 3 días), hacen que no se puedan considerar como analgésicos de pri- mera línea. Sólo se debería considerar su uso ante el fracaso de los analgésicos según la escala de la Organización Mundial de la Salud(OMS) y otros procedimientos como la radioterapia antiálgica o la cirugía (15).

Según las recomendaciones de la American Society of Clinical Oncology (ASCO), la indicaciones aceptadas para el empleo bifosfonatos incluyen la hipercalcemia tumoral y los eventos óseos asociados al cáncer de mama y mieloma múltiple $(18,19)$.

Aunque existen resultados satisfactorios sobre las metastásis óseas de cáncer de próstata y de otros tumores sólidos, el empleo de los bifosfonatos no está claramente indicado en estos casos.

PAPEL DE LOS BIFOSFONATOS EN CÁNCER DE MAMA METASTÁSICO CON LESIONES OSTEOLÍTICAS (ASCO 2003)

En el año 2003 los expertos convocados por la ASCO establecieron unas serie de recomendaciones acerca del uso de los bifosfonatos en el cáncer de mama (18):

1. En los casos de cáncer de mama con lesiones osteolíticas en la radiografías simples el empleo de zolendronato (4 $\mathrm{mg}$ en $15 \mathrm{~min}$.) o pamidronato (90 $\mathrm{mg}$ en 2 horas) por vía intravenosa cada 3-4 semanas es recomendable. No existe suficiente evidencia que indique que ninguno de los dos sea superior al otro (20). La presencia o ausencia del dolor óseo no debería de ser un factor a tener en cuenta a la hora de iniciar los bifosfonatos.

2. En los casos en los que hay un rastreo óseo patológico, pero las radiografías simples normales, se debe realizar TC o RM ósea; y si éstas últimas presentan destrucción ósea, el empleo de los bifosfonatos es razonable.

3. Si hay un rastreo óseo patológico, pero tanto las radiografías óseas como TC o RM óseas son normales, no está recomendado el uso de los bifosfonatos.

4. En diversos ensayos clínicos se ha valorado la utilidad de los bifosfonatos en la adyuvancia de cáncer de mama $(21,22)$; sin embargo los expertos de la ASCO no recomiendan su uso fuera de los ensayos clínicos.

Si la creatinina es igual o inferior a $3 \mathrm{mgdl}$ no es necesario modificaciones en la dosis, ni en el tiempo de infusión, ni en el intervalo de su administración. El tratamiento con bifosfonatos debe mantenerse hasta que aparezca un empeoramiento significativo en el performance status del paciente.

Además la ASCO recomienda monitorizar de manera periódica los niveles de creatinina, electrólitos, magnesio, calcio, fósforo y de hemoglobina.

PREVENCIÓN Y TRATAMIENTO DE LA OSTEOPOROSIS ASOCIADO AL CÁNCER DE MAMA

Los pacientes con cáncer de mama tienen por lo general alto riesgo de desarrollar osteoporosis, ya sea por la edad en la que se diagnostica el cáncer de mama o por el efecto del tratamiento (18); motivo por el cual el oncólogo médico debe evaluar correctamente la salud ósea de las mujeres con cáncer de mama. Los factores de alto riesgo de padecer osteoporosis se resumen en la tabla I. 


\section{TABLA I}

\section{FACTORES DE ALTO RIESGO DE OSTEOPOROSIS}

1. Edad igual o mayor de 65 años

2. Edad entre 60 y 64 años y al menos uno de los siguientes:

-Antecedentes familiares de osteoporosis

-Peso corporal $<70 \mathrm{~kg}$

- Antecedente de fractura no traumática

3. Mujer posmenopáusica en tratamiento con inhibidores de la aromatasa

4. Mujer premenopáusica que presenta menopausia precoz por el tratamiento u otros motivos:

-Ablación ovárica por cirugía, radioterapia, quimioterapia u hormonoterapia

Entre los fármacos que se emplean en el tratamiento de la osteoporosis se debe de distinguir dos grandes grupos:

-Fármacos que disminuyen la incidencia de fracturas vertebrales y extravertebrales: bifosfonatos (risedronato, alendronato, zolendronato) y PTH (teriparitide).
-Fármacos que disminuyen solamente la incidencia de fracturas vertebrales: raloxifeno y calcitonina.

El manejo de la osteoporosis en las mujeres con cáncer de mama, según los distintos grupos de riesgo, se detalla en la figura 1 .

\section{BIFOSFONATOS Y MIELOMA MÚLTIPLE}

Los bifosfonatos por vía intravenosa (zolendronato $4 \mathrm{mg}$ en 15 minutos y pamidronato $90 \mathrm{mg}$ en 2 horas) administrados de manera periódica, son de alta utilidad en el tratamiento del mieloma múltiple (23), con reducción de riesgo fracturas patológicas y de la hipercalcemia tumoral, así como disminuyendo el dolor óseo secundario a las lesiones osteolíticas y proporcionando un efecto antimielomatoso (13).

En los casos de gamapatía monoclonal de significado incierto, plasmocitoma solitario sin lesiones osteolíticas o mieloma múltiple asintomático (quiescente o smoldering) no está indicado iniciar el tratamiento con bifosfonatos. Sin embargo, en los casos de mieloma múltiple sintomático con lesiones osteolíticas el empleo de bifosfonatos endovenosos estaría recomendado; y en los casos donde se evidencia una

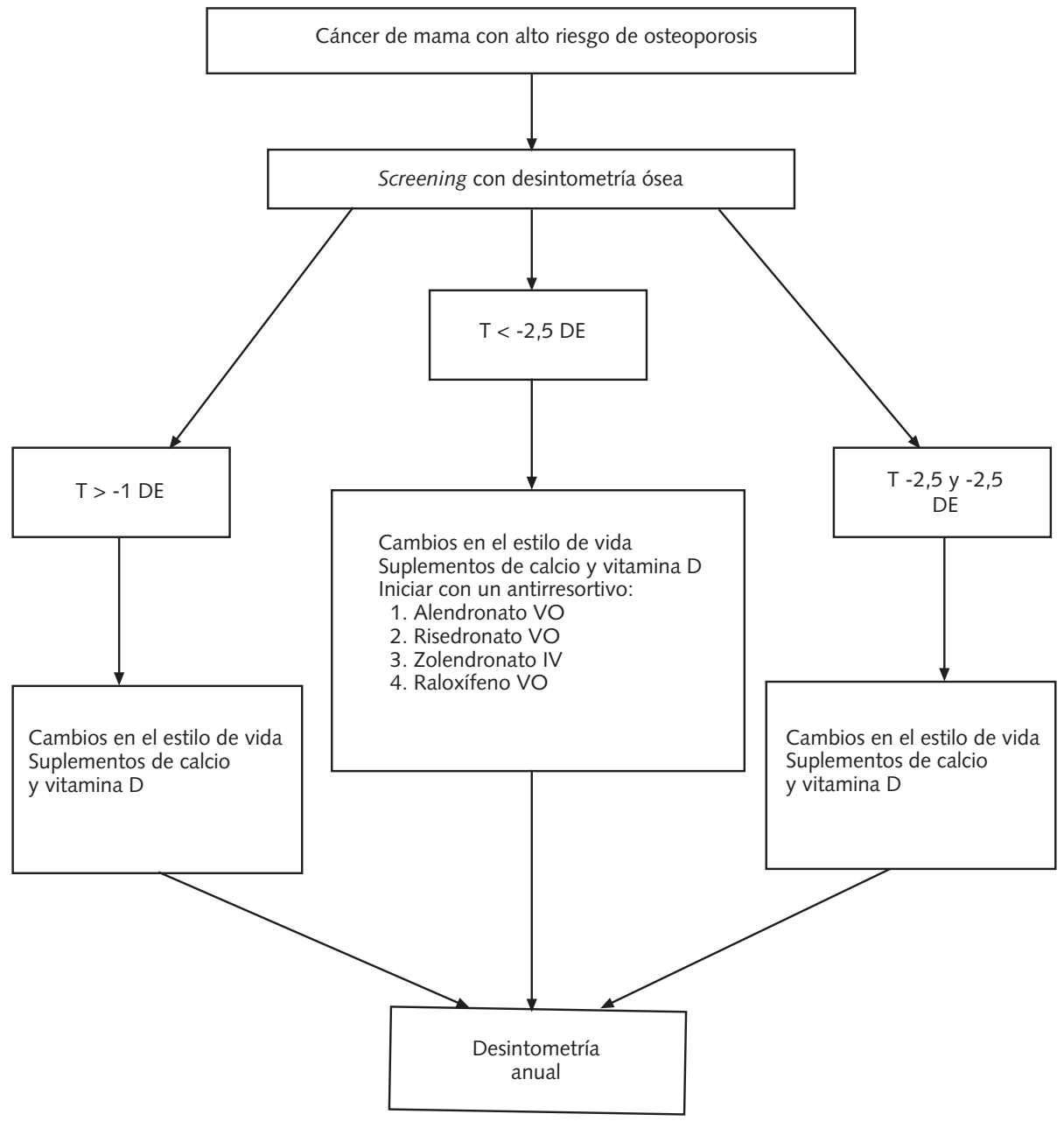

VO: vía oral; IV: intravenoso; DE: desviaciones estándar; T: índice T de la desintometría ósea.

Fig. 1. Estrategia terapéutica ante cáncer de mama con alto riesgo de osteoporosis. 
osteopenia en radiografías simples o en la densitometría ósea el empleo de estos fármacos se considera razonable (19).

También se recomienda una monitorización periódica cada 3-6 meses de la función renal mediante la determinación de aclaramiento de creatinina, creatinina sérica, iones con calcio y fósforo y albuminuria.

\section{Bibliografía}

1. Fleisch H. Biphosphonates: mechanisms of action. Endocr Rev 1998; 19: 80-100.

2. Cheer SM, Noble S. Zolendronic acid. Drugs 2001; 61: 799-805.

3. Cyer B, Bauer DC. Oral biphosphonates and upper gastrointestinal tract problems: what is the evidence? Mayo Clin Proc 2002; 77: 1031-43.

4. Pelayo M, Agra Y. Bifosfonatos en la prevención de la osteoporosis de mujeres posmenopáusicas con baja masa ósea. Med Clin (Barc) 2004; 122: 304-10.

5. Delmas PD, Meunier PJ. The managment of Paget's disease of bone. N Engl J Med 1997; 336: 558-66.

6. Watts NB. Treatment of osteoporosis with biphosphonates. Rheum Dis Clin North Am 2001; 27: 197-214.

7. Rodino MA, Shane E. Osteoporosis after organ transplantation. Am J Med 1998; 104: 459-69.

8. Falk MJ, Heeger S, Lynch KA, Decaro KR, Bohach D, Gibson Ks, et al. Intravenous biphosphonate therapy in children with osteogenesis imperfecta. Pediatrics 2003; 111: 573-8

9. Berenson JR. Treatment of hypercalcemia of malignancy with biphosphonates. Semin Oncol 2002; 29: 12-8.

10. Coleman RE. Biphosphonates for the prevention of bone metastases. Semin Oncol 2002; 29: 43-9.

11. Ralston SH, Gallagher SJ, Patel U, Campbell J, Boyle IT. Cancer-associated hypercalcemia: morbidity and mortality: clinical experience in 126 treated patients. Ann Intern Med 1990; 112: 499-504.

12. Stewart AF. Hypercalcemia associated with Cancer. N Engl J Med 2005;352: 373-379.

13. Roodman GD. Mechanisms of bone metastasis. N Engl J Med 2004; 350: 1655-64.

14. Horwitz MJ, Stewart AF. Humoral hypercalcemia of malignancy. In: Favus MF,ed. Primer on the metabolic bone diseases and disorders of
En los casos en los cuales se detecte un deterioro inexplicable de la función renal, de los electrólitos y/o proteinuria, se debe de interrumpir temporalmente la administración de los bifosfonatos, hasta la normalización de dichos parámetros, para decidir con posterioridad su reintroducción.

mineral metabolism. 5th ed. Washington D.C. Am Soc Bone Min Res, 2003: 246-50.

15. Hernández JL, Riancho JA, González J. Bifosfonatos intravenosos. Med Clin (Barc) 2005; 124: 384-54

16. Major P, Lortholary A, Hon J. Zolendronic acid is superior to pamidronate in the treatment of hypercalcemia of malignancy: a pooled analysis of two randomized controlled clinical trials. J Clin Oncol 2001; 19: 55867.

17. Wong R, Wiffen PJ. Biphosphonates for the relief of pain secondary to bone metastases. Cochrane Database Syst Rev 2002; 2: CD002068.

18. Hillner BE, Ingle JN, Chlebowski RT, Gralow J, Yee GC, Janjan NA et al. American Society of Clinical Oncology 2003 Update on the Role of Bisphosphonates and Bone Health Issues in Women with breast cancer. J Clin Oncol 2003; 21: 4042-4057.

19. Berenson JR, Hillner BE, Kyle RA, Anderson K, Lipton A, Yee GC et al. American Society of Clinical Oncology practice guidelines: The role of bisphosphonates in multiple myeloma. J Clin Oncol 2002; 17: 37193736.

20. Rosen LS, Gordon D, Kaminski M. Zolendronic acid versus pamidronate in the treatment of skeletal metastases in patients with breast cancer or osteolytic lesions of multiple myeloma: a phase III, double-blind, comparative trial. Cancer J 2001; 7: 377-387.

21. Saarto T, Blomqvist C, Virkkunen P. No reduction of bony metastases with adjuvant clodronate treatment in node-positive breast cancer patients. J Clin Oncol 2001; 19: 10-17.

22. Powles T, Paterson S. Randomized, placebo-controlled trial of clodronate in patients with primary operable breast cancer. J Clin Oncol 2002; 20: 3219-3224.

23. Kyle RA, Rajkumar SV. Multiple myeloma. N Engl J Med 2004; 351 : 1860-73. 